arXiv:0802.1020

IFUP-TH/2008-02

TIT/HEP-579

DAMTP-2008-8

\title{
Constructing Non-Abelian Vortices with Arbitrary Gauge Groups
}

\author{
Minoru Eto ${ }^{1,2}$, Toshiaki Fujimori ${ }^{3}$, Sven Bjarke Gudnason ${ }^{1,2}$, Kenichi Konishi ${ }^{1,2}$, \\ Muneto Nitta ${ }^{4}$, Keisuke Ohashi ${ }^{5}$ and Walter Vinci ${ }^{1,2}$ \\ 1 Department of Physics, University of Pisa, Largo Pontecorvo, 3, Ed. C, 56127 Pisa, \\ Italy \\ ${ }^{2}$ INFN, Sezione di Pisa, Largo Pontecorvo, 3, Ed. C, 56127 Pisa, Italy \\ 3 Department of Physics, Tokyo Institute of Technology, Tokyo 152-8551, Japan \\ 4 Department of Physics, Keio University, Hiyoshi, Yokohama, Kanagawa 223-8521, \\ Japan \\ ${ }^{5}$ Department of Applied Mathematics and Theoretical Physics, University of Cambridge, \\ CB3 $0 W A, U K$
}

\begin{abstract}
We construct the general vortex solution in the fully-Higgsed, color-flavor locked vacuum of a non-Abelian gauge theory, where the gauge group is taken to be the product of an arbitrary simple group and $U(1)$, with a Fayet-Iliopoulos term. The vortex moduli space is determined.
\end{abstract}

PACS: 11.27.+d, 11.30.Pb, 11.25.-w, 12.10.-g 


\section{Introduction}

Vortices play important roles in various areas of physics from condensed matter physics such as superconductors, superfluids, quantum Hall effects to particle physics [1 and cosmology [2]. Recently there has been a significant progress in the understanding of non-Abelian vortices in the color-flavor locked vacuum of $S U(N) \times U(1)$ gauge theories [3, 4]. Unlike Abelian vortices [5], they carry orientational moduli in the internal space, in addition to the usual position moduli. The most general Bogomol'nyi-Prasad-Sommerfield (BPS) vortex solutions and their moduli space have been found [6, 7] and the dynamics of two colliding vortices studied [8]. Though these and many other interesting features have been extensively explored [6]-[15], most studies so far have been restricted to the gauge group $S U(N) \times U(1)$, with a few but notable exceptions [11], [16].

We present here a simple framework for writing the most general non-Abelian BPS vortex solutions in theories with an arbitrary gauge group of the type $G=G^{\prime} \times U(1)$. For concreteness we take $G^{\prime}$ to be a simple Lie group, but the method can be easily generalized to non-simple groups. The cases of classical groups $G^{\prime}=S U, S O, U S p$ will be worked out in detail. Various new physical results seem to follow from our study, even though here we limit ourselves to the main results only. Fuller account will be given elsewhere[17].

\section{Model and BPS Vortex Equations}

We focus our attention on the classical Lie groups $G^{\prime}=S U(N), S O(N)$ and $U S p(2 M)$, leaving the exceptional groups to a short discussion at the end. For $G^{\prime}=S O(N), U S p(2 M)$ their group elements are embedded into $S U(N)(N=2 M$ for $U S p)$ by constraints of the form, $U^{T} J U=J$, where $J$ is the rank-2 invariant tensor

$$
J=\left(\begin{array}{cc}
\mathbf{0}_{M} & \mathbf{1}_{M} \\
\epsilon \mathbf{1}_{M} & \mathbf{0}_{M}
\end{array}\right), \quad\left(\begin{array}{ccc}
\mathbf{0}_{M} & \mathbf{1}_{M} & 0 \\
\mathbf{1}_{M} & \mathbf{0}_{M} & 0 \\
0 & 0 & 1
\end{array}\right)
$$

where $\epsilon=+1$ for $S O(2 M)$, while $\epsilon=-1$ for $U S p(2 M)$; the second matrix is for $S O(2 M+$ 1). Apart from the gauge bosons $W_{\mu}=W_{\mu}^{0} t^{0}+W_{\mu}^{a} t^{a}$ the matter content of the model consists of $N$ flavors of Higgs scalar fields in the fundamental representation, with a common $U(1)$ charge, written as a color-flavor mixed $N \times N$ matrix $H . t^{0}$ and $t^{a}$ denote the generators of $U(1)$ and $G^{\prime}$ normalized as

$$
t^{0}=\frac{\mathbf{1}_{N}}{\sqrt{2 N}}, \quad \operatorname{tr}\left(t^{a} t^{b}\right)=\frac{\delta^{a b}}{2}
$$


The Lagrangian is given by

$$
\begin{aligned}
\mathcal{L}= & -\frac{1}{4 e^{2}} F_{\mu \nu}^{0} F^{0 \mu \nu}-\frac{1}{4 g^{2}} F_{\mu \nu}^{a} F^{a \mu \nu}+\left(\mathcal{D}_{\mu} H_{A}\right)^{\dagger} \mathcal{D}^{\mu} H_{A} \\
& -\frac{e^{2}}{2}\left|H_{A}^{\dagger} t^{0} H_{A}-\frac{v^{2}}{\sqrt{2 N}}\right|^{2}-\frac{g^{2}}{2}\left|H_{A}^{\dagger} t^{a} H_{A}\right|^{2},
\end{aligned}
$$

where $\mathcal{D}_{\mu} H=\left(\partial_{\mu}+i W_{\mu}\right) H, e$ and $g$ are the gauge coupling constants for $U(1)$ and $G^{\prime}$, respectively, and $A$ is the flavor index. The flavor symmetry of the model is $S U(N)_{\mathrm{F}}$. Though our discussion concerns mainly the bosonic system, (3), the model is really to be considered as the (truncated) bosonic sector of the corresponding $\mathcal{N}=2$ supersymmetric gauge theory, which explains the particular form of the potential, ensuring at the same time its stability against radiative corrections. The Bogomol'nyi completion for static, $x^{3}$ independent configurations

$$
\begin{aligned}
T=\int d^{2} x\left[\frac{1}{2 e^{2}}\left|F_{12}^{0}-e^{2}\left(H_{A}^{\dagger} t^{0} H_{A}-\frac{v^{2}}{\sqrt{2 N}}\right)\right|^{2}\right. \\
\left.\quad+4\left|\mathcal{D}_{\bar{z}} H\right|^{2}+\frac{1}{2 g^{2}}\left|F_{12}^{a}-g^{2} H_{A}^{\dagger} t^{a} H_{A}\right|^{2}-\frac{v^{2}}{\sqrt{2 N}} F_{12}^{0}\right] \\
\geq-\frac{v^{2}}{\sqrt{2 N}} \int d^{2} x F_{12}^{0}
\end{aligned}
$$

yields the BPS vortex equations

$$
\begin{aligned}
\mathcal{D}_{\bar{z}} H & =0, \\
F_{12}^{0}-\frac{e^{2}}{\sqrt{2 N}}\left(\operatorname{tr}\left(H H^{\dagger}\right)-v^{2}\right) & =0, \\
F_{12}^{a} t^{a}-\frac{g^{2}}{4}\left(H H^{\dagger}-J^{\dagger}\left(H H^{\dagger}\right)^{T} J\right) & =0,
\end{aligned}
$$

for the groups $G^{\prime}=S O(N), U S p(2 M)$, where a complex coordinate $z \equiv x^{1}+i x^{2}$ has been introduced. Equation (7) reads for $G^{\prime}=S U(N)$ instead:

$$
F_{12}^{a} t^{a}-\frac{g^{2}}{2}\left[H H^{\dagger}-\frac{\mathbf{1}_{N}}{N} \operatorname{tr}\left(H H^{\dagger}\right)\right]=0 .
$$

\section{Solving the BPS Vortex Equations}

Let us choose the fully Higgsed, color-flavor locked vacuum: $\langle H\rangle=\frac{v}{\sqrt{N}} \mathbf{1}_{N}$. The $G^{\prime} \times$ $U(1) \times S U(N)_{\mathrm{F}}$ invariance of the theory is broken to the global color-flavor diagonal 
$G_{\mathrm{C}+\mathrm{F}}^{\prime}$. Introduce an $N$ by $N$ matrix $S(z, \bar{z})$ taking a value in the complexification $G^{\mathbb{C}}$ of $G$,

$$
S(z, \bar{z})=S_{e}(z, \bar{z}) S^{\prime}(z, \bar{z})
$$

with $S_{e} \in U(1)^{\mathbb{C}} \simeq \mathbb{C}^{*}$ and $S^{\prime} \in G^{\prime \mathbb{C}}$. The gauge fields can be taken to be equal to the Maurer-Cartan form:

$$
\begin{aligned}
\left(W_{1}^{0}+i W_{2}^{0}\right) t^{0} & =-2 i S_{e}^{-1} \bar{\partial} S_{e}, \\
\left(W_{1}^{a}+i W_{2}^{a}\right) t^{a} & =-2 i S^{-1} \bar{\partial} S^{\prime} .
\end{aligned}
$$

The first of the BPS equations (5) can then be solved by

$$
H=S^{-1} H_{0}(z)=S_{e}^{-1} S^{-1} H_{0}(z),
$$

where $H_{0}(z)$ is a matrix whose elements are holomorphic in $z . H_{0}(z)$ will be called moduli matrix [12], as all moduli parameters are encoded in it (see below). $H_{0}(z)$ is defined up to equivalence relations of the form

$$
\left(H_{0}, S\right) \sim V_{e} V^{\prime}(z)\left(H_{0}, S\right), \quad V^{\prime}(z)^{T} J V^{\prime}(z)=J .
$$

By introducing $N \times N$ matrices $\Omega_{0} \equiv H_{0} H_{0}^{\dagger}$ and

$$
\Omega_{e} \equiv S_{e} S_{e}^{\dagger} \equiv e^{\psi \mathbf{1}_{2 N}} \in U(1)^{\mathbb{C}}, \quad \Omega^{\prime} \equiv S^{\prime} S^{\prime \dagger} \in G^{\prime \mathbb{C}},
$$

the BPS equations (6), (77) can be cast into the form:

$$
\begin{gathered}
\bar{\partial} \partial \psi=-\frac{e^{2}}{4 N}\left(\operatorname{tr}\left(\Omega_{0} \Omega^{\prime-1}\right) e^{-\psi}-v^{2}\right), \\
\bar{\partial}\left(\Omega^{\prime} \partial \Omega^{\prime-1}\right)=\frac{g^{2}}{8}\left(\Omega_{0} \Omega^{\prime-1}-J^{\dagger}\left(\Omega_{0} \Omega^{\prime-1}\right)^{T} J\right) e^{-\psi},
\end{gathered}
$$

which we denote the master equations. The boundary conditions $\operatorname{are} \operatorname{tr}\left(\Omega_{0} \Omega^{\prime-1}\right) e^{-\psi}=v^{2}$ and $\Omega_{0} \Omega^{\prime-1}=J^{\dagger}\left(\Omega_{0} \Omega^{\prime-1}\right)^{T} J$. We assume the existence and uniqueness for the solutions to these equations. There are at least two justifications for this. One is the fact that in the strong coupling limit $(e, g \rightarrow \infty)$ these can be algebraically and uniquely solved. The other relies on the index theorem: the number of the moduli parameters encoded in $H_{0}$ coincide with that obtained from the index theorem [17].

The tension of the BPS vortices can be written as

$$
T=-\frac{v^{2}}{\sqrt{2 N}} \int d^{2} x F_{12}^{0}=2 v^{2} \int d^{2} x \bar{\partial} \partial \psi .
$$

The asymptotic behavior

$$
S_{e} \sim|z|^{\nu} \text { for }|z| \rightarrow \infty
$$


then determines the tension

$$
T=2 \pi v^{2} \nu,
$$

a rational number $\nu(>0)$ being the $U(1)$ winding number. $\nu$ will be found to be quantized in half-integers $(\nu=k / 2)$ for the groups $G^{\prime}=S O(2 M), U S p(2 M)$ with $k \in \mathbb{Z}_{+} ; \nu=k$ (integers) for $G^{\prime}=S O(2 M+1)$; finally $\nu=k / N$ for $G^{\prime}=S U(N)$, as is well known. The integer $k$ denotes the vortex number: $k=1$ corresponds to the minimal vortex in all cases.

The key idea of this Letter, which enables us to extend the moduli-matrix formalism to general gauge groups, is to consider the holomorphic invariants $I_{G^{\prime}}^{i}(H)$ made of $H$, which are invariant under $G^{\prime \mathbb{C}}$, with $i$ labeling them. If the $U(1)$ charge of the $i$-th invariant $I_{G^{\prime}}^{i}(H)$ is $n_{i}$, the following relation

$$
I_{G^{\prime}}^{i}(H)=I_{G^{\prime}}^{i}\left(S_{e}^{-1} S^{\prime-1} H_{0}\right)=S_{e}^{-n_{i}} I_{G^{\prime}}^{i}\left(H_{0}(z)\right),
$$

holds. If the boundary condition is given by

$$
\left.I_{G^{\prime}}^{i}(H)\right|_{|z| \rightarrow \infty}=I_{\mathrm{vev}}^{i} e^{i \nu n_{i} \theta},
$$

where $\nu n_{i}$ is the number of zeros of $I_{G^{\prime}}^{i}$, it follows that

$$
I_{G^{\prime}}^{i}\left(H_{0}\right)=S_{e}^{n_{i}} I_{G^{\prime}}^{i}(H) \sim I_{\mathrm{vev}}^{i} z^{\nu n_{i}}, \quad|z| \rightarrow \infty .
$$

As $I_{G^{\prime}}^{i}\left(H_{0}(z)\right)$ are holomorphic, the above condition implies that $I_{G^{\prime}}^{i}\left(H_{0}(z)\right)$ are polynomials in $z$. We find that $\nu n_{i}$ must be a positive integer for all $i$ :

$$
\nu n_{i} \in \mathbb{Z}_{+} \quad \rightarrow \quad \nu=k / n_{0}, \quad k \in \mathbb{Z}_{+},
$$

where $(\mathrm{GCD}=$ the greatest common divisor $)$

$$
n_{0} \equiv \operatorname{GCD}\left\{n_{i} \mid I_{\mathrm{vev}}^{i} \neq 0\right\} \text {. }
$$

Note that a $U(1)$ gauge transformation $e^{2 \pi i / n_{0}}$ leaves invariant $I_{G^{\prime}}^{i}(H)$ :

$$
I_{G^{\prime}}^{i}\left(H^{\prime}\right)=e^{2 \pi i n_{i} / n_{0}} I_{G^{\prime}}^{i}(H)=I_{G^{\prime}}^{i}(H):
$$

the phase rotation $e^{2 \pi i / n_{0}} \in \mathbb{Z}_{n_{0}}$ changes no physics, and the true gauge group is thus

$$
G=U(1) \times G^{\prime} / \mathbb{Z}_{n_{0}} .
$$

where $\mathbb{Z}_{n_{0}}$ is the center of $G^{\prime}$. A simple homotopy argument tells us that $1 / n_{0}$ is the $U(1)$ winding for the minimal $(k=1)$ vortex configuration. Finally, for a given $k$ the following important relations hold

$$
I_{G^{\prime}}^{i}\left(H_{0}\right)=I_{\mathrm{vev}}^{i} z^{k n_{i} / n_{0}}+\mathcal{O}\left(z^{k n_{i} / n_{0}-1}\right),
$$


which imply nontrivial constraints on $H_{0}(z)$.

The explicit form of the constraints follows from this general discussion. For $G^{\prime}=$ $S U(N)$ (with $N$ flavors), there exists only one invariant

$$
I_{S U}=\operatorname{det}(H),
$$

with charge $N$. Thus the minimal winding $\left(1 / n_{0}\right)$ is equal to $1 / N$ and the condition for $k$ vortices is given by:

$$
A_{N-1}: \operatorname{det} H_{0}(z)=z^{k}+\mathcal{O}\left(z^{k-1}\right), \quad \nu=k / N .
$$

For $G^{\prime}=S O(N), U S p(2 M)$, there are $N(N \pm 1) / 2$ invariants

$$
\left(I_{S O, U S P}\right)^{r}{ }_{s}=\left(H^{\mathrm{T}} J H\right)^{r}{ }_{s}, \quad 1 \leq r \leq s \leq N,
$$

in addition to (27). The constraints are:

$$
\begin{aligned}
& C_{M}, D_{M}: H_{0}^{T}(z) J H_{0}(z)=z^{k} J+\mathcal{O}\left(z^{k-1}\right), \quad \nu=k / 2, \\
& B_{M}: H_{0}^{T}(z) J H_{0}(z)=z^{2 k} J+\mathcal{O}\left(z^{2 k-1}\right), \nu=k,
\end{aligned}
$$

for $G^{\prime}=S O(2 M), U S p(2 M)$ and $S O(2 M+1)$, respectively. As anticipated, vortices in the $S O(2 M+1)$ model have integer $U(1)$ windings [11.

Explicitly, the minimal vortices in $S U(N)$ and $S O(2 M)$ or $U S p(2 M)$ theories are given respectively by the moduli matrices:

$$
H_{0}=\left(\begin{array}{cc}
z-a & 0 \\
\mathbf{b} & \mathbf{1}_{N-1}
\end{array}\right),\left(\begin{array}{cc}
z \mathbf{1}_{M}-\mathbf{A} & \mathbf{C}_{S / A} \\
\mathbf{B}_{A / S} & \mathbf{1}_{M}
\end{array}\right)
$$

The moduli parameters are all complex. For $S U(N)$, $a$ is just a number; b is a column vector. For $S O(2 M)$ or $U S p(2 M)$, the matrix $\mathbf{C}_{S / A}$ for instance is symmetric or antisymmetric, respectively. And vice versa for B. Moduli matrices for $S O(2 M+1)$ as well as those for $k=2$ vortices in $S U, S O, U S p$ theories, will be given explicitly [17].

The index theorem gives the complex dimension of the moduli space

$$
\operatorname{dim}_{\mathbb{C}}\left(\mathcal{M}_{G^{\prime}, k}\right)=\frac{k N^{2}}{n_{0}} .
$$

This was obtained in [3] for $S U(N)$; a proof in other cases will be reported elsewhere [17]. In all cases studied we have checked that the dimension of the moduli space inferred from our moduli matrices agrees with the one given in Eq. (32).

Except for the $S U(N)$ case, our model has a non-trivial Higgs branch (flat directions). The color-flavor locked vacuum $\langle H\rangle \propto \mathbf{1}_{N}$ is just one of the possible (albeit the most symmetric) choices for the vacuum; our discussion can readily be generalized to a generic vacuum on the Higgs branch. This fact, however, implies that our non-Abelian vortices have "semilocal" moduli (see Achucarro et. al. [1]), even for $N_{f}=N$. In contrast to the Abelian or $S U(N)$ cases, moreover, they exhibit new, interesting phenomena such as "fractional" vortices [17]. 


\section{Local (ANO-like) Vortices}

For various considerations, we are interested in knowing which of the moduli parameters describe the so-called local vortices, the ANO-type vortices with exponential tails. To identify these, let us first consider generic points in the moduli space. In the strong coupling limit our theory reduces to a nonlinear sigma model, with the (classical) vacuum moduli $\mathcal{M}_{\text {vac }}$ as its target space. In such a limit, semilocal vortices with non-zero size moduli reduce to the so-called sigma model lumps. The local vortices on the other hand shrink to singular configurations. It is well-known that lumps are characterized by $\pi_{2}\left(\mathcal{M}_{\text {vac }}\right)$ with a wrapping around a 2 -cycle inside $\mathcal{M}_{\text {vac }}$. Even at a finite gauge coupling, asymptotic configurations of semilocal vortices can be well approximated by lumps.

Now the moduli space of vacua $\mathcal{M}_{\text {vac }}$ in supersymmetric models is parametrized by holomorphic invariants $I_{G}^{I}(H)(I=1,2, \ldots)$ of the complexified gauge group $G^{\mathbb{C}}$ [18. In our case, $G=G^{\prime} \times U(1)$, with the common $U(1)$ charge of the scalar fields $H$, all the $G^{\mathbb{C}}$ invariants $I_{G}^{I}(H)$ can be written using the $G^{\mathbb{C}}$ invariants $I_{G^{\prime}}^{i}(H)$. For instance from $I_{G^{\prime}}^{i}$ and $I_{G^{\prime}}^{j}$ with $n_{i}=n_{j}$, one can construct

$$
I_{G}^{(i, j)}(H) \equiv \frac{I_{G^{\prime}}^{i}(H)}{I_{G^{\prime}}^{j}(H)}=\frac{I_{G^{\prime}}^{i}\left(H_{0}(z)\right)}{I_{G^{\prime}}^{j}\left(H_{0}(z)\right)},
$$

where use was made of (19). The last line defines, so called, (generalized) rational maps. This observation allows us to define local vortices. While asymptotic region of semilocal vortices are mapped to some domain of $\mathcal{M}_{\mathrm{vac}}$, those around the local vortices are mapped into a single point. Therefore, all the $G^{\mathbb{C}}$ invariants $I_{G}^{I}(H)$ must be constant for the latter. All the $I_{G^{\prime}}(H)$ 's have zeros at the vortex positions and winding around them as seen in (20). These facts, together with (33), imply that all $I_{G^{\prime}}^{i}\left(H_{0}(z)\right)$ 's with the same $n_{i}$ must have common zeros:

$$
I_{G^{\prime}}^{i}\left(H_{0, \text { local }}\right)=\left[\prod_{\ell=1}^{k}\left(z-z_{0 \ell}\right)\right]^{n_{i} / n_{0}} I_{\mathrm{vev}}^{i} .
$$

For $G^{\prime}=S O(2 M), U S p(2 M)$ with $I_{S O, U S p}$ of (29) we find that the condition for vortices to be of local type is

$$
H_{0, \text { local }}^{T}(z) J H_{0, \text { local }}(z)=\prod_{\ell=1}^{k}\left(z-z_{0 \ell}\right) J .
$$

Let us now discuss a few concrete examples. The general solution for the minimal vortex (31) for $G^{\prime}=\{S U(N), S O(2 M), U S p(2 M)\}$ is reduced to a local vortex if we restrict it to be of the form:

$$
H_{0, \text { local }}=\left(\begin{array}{cc}
z-a & 0 \\
\mathbf{b} & \mathbf{1}_{N-1}
\end{array}\right),\left(\begin{array}{cc}
(z-a) \mathbf{1}_{M} & 0 \\
\mathbf{B}_{A / S} & \mathbf{1}_{M}
\end{array}\right) .
$$


The vortex position is given by $a$. $\mathbf{b}$ for $S U(N)$ and $\mathbf{B}_{A / S}$ for $S O(2 M)$ or $U S p(2 M)$ encode the Nambu-Goldstone modes associated with the breaking of the color-flavor symmetry by the vortex $G_{\mathrm{C}+\mathrm{F}}^{\prime} \rightarrow H_{G^{\prime}}$. The moduli spaces are direct products of a complex number and the Hermitian symmetric spaces

$$
\mathcal{M}_{G^{\prime}, k=1}^{\text {local }} \simeq \mathbb{C} \times G_{\mathrm{C}+\mathrm{F}}^{\prime} / H_{G^{\prime}},
$$

$H_{S U(N)}=S U(N-1) \times U(1)$ while $H_{S O(2 M), U S p(2 M)}=U(M)$. The results for $S U(N)$ and $S O(2 M)$ are well-known [3, 4, 11. The matrices (36) describe just one patch of the moduli space. In order to define the manifold globally we need a sufficient number of patches. The number of patches is $N$ for $G^{\prime}=S U(N)$ and $2^{M}$ for $G^{\prime}=S O(2 M), U S p(2 M)$. The transition functions correspond to the $V$-equivalence relations [6, 17. In the case of $G^{\prime}=$ $S O(2 M)$, the patches are given by permutation of the $i$-th and the $(M+i)$-th columns in (36). We find that no regular transition functions connect the odd and even permutations (patches), hence the moduli space consists of two disconnected copies of $S O(2 M) / U(M)$ [11]. The complex dimensions of the moduli spaces are $\operatorname{dim}_{\mathbb{C}} \mathcal{M}_{S O(2 M), k=1}^{\text {local }}=\frac{1}{2} M(M-1)+1$ and $\operatorname{dim}_{\mathbb{C}} \mathcal{M}_{U S p(2 M), k=1}^{\text {local }}=\frac{1}{2} M(M+1)+1$.

\section{$5 \quad$ Exceptional groups}

$\boldsymbol{E}_{6}$ : There is a rank-3 symmetric tensor: $\Gamma_{i j k}$. The conditions on the moduli matrix is

$$
\Gamma_{i_{1} i_{2} i_{3}}\left(H_{0}\right)^{i_{1}}{ }_{j_{1}}\left(H_{0}\right)^{i_{2}}{ }_{j_{2}}\left(H_{0}\right)^{i_{3}}{ }_{j_{3}} \sim \Gamma_{j_{1} j_{2} j_{3}} z^{k},
$$

and the $U(1)$ winding number is quantized as $\nu=k / 3$.

$\boldsymbol{E}_{7}$ : There are 2 invariant tensors: $d_{i j k l}$ and $f_{i j}$ respectively of rank 4 and 2 . The moduli matrix is constrained as:

$$
\begin{aligned}
& d_{i_{1} i_{2} i_{3} i_{4}}\left(H_{0}\right)^{i_{1}}{ }_{j_{1}}\left(H_{0}\right)^{i_{2}}{ }_{j_{2}}\left(H_{0}\right)^{i_{3} j_{3}}\left(H_{0}\right)^{i_{4}}{ }_{j_{4}} \sim d_{j_{1} j_{2} j_{3} j_{4}} z^{2 k}, \\
& f_{i_{1} i_{2}}\left(H_{0}\right)^{i_{1}}{ }_{j_{1}}\left(H_{0}\right)^{i_{2}}{ }_{j_{2}} \sim f_{j_{1} j_{2}} z^{k},
\end{aligned}
$$

and the vortices are quantized in half integers: $\nu=k / 2$.

$\boldsymbol{G}_{2}, \boldsymbol{F}_{4}, \boldsymbol{E}_{8}$ : See Table 1 for the list of the invariant tensors and the winding numbers.

\section{Conclusion}

We have thus given all the necessary tools to construct vortex solutions in the color-flavor locked vacuum of a non-Abelian gauge theory with gauge group $G=G^{\prime} \times U(1)$ where $G^{\prime}$ is an arbitrary simple group, coupled to Higgs fields in the fundamental representation. Our method can be extended to other BPS solitons such as domain walls, monopoles and instantons, and hopefully opens powerful new windows for their investigation. 


\begin{tabular}{|c|c|c|c|c|c|c|c|c|}
\hline$G^{\prime}$ & $A_{N-1}$ & $B_{M}$ & $C_{M}, D_{M}$ & $E_{6}$ & $E_{7}$ & $E_{8}$ & $F_{4}$ & $G_{2}$ \\
\hline$R$ & $N$ & $2 M+1$ & $2 M$ & 27 & 56 & 248 & 26 & 7 \\
\hline rank inv & - & 2 & 2 & 3 & 2,4 & $2,3,8$ & 2,3 & 2,3 \\
\hline$n_{0}$ & $N$ & 1 & 2 & 3 & 2 & 1 & 1 & 1 \\
\hline
\end{tabular}

Table 1: The dimension of the fundamental representation $(R)$, the rank of the other invariants [19] and the minimal tension $\nu=1 / n_{0}$ i.e. the center $\mathbb{Z}_{n_{0}}$ of $G^{\prime}$. The determinant of the $R \times R$ matrix gives one invariant with charge, $\operatorname{dim} R$.

\section{Acknowledgment}

The authors thank Luca Ferretti, Giacomo Marmorini and David Tong for useful discussions. T.F, M.N. and K.O. would like to thank theoretical high energy physics group at Pisa and INFN for their hospitality. The work of T.F. is supported by the Research Fellowships of the Japan Society for the Promotion of Science for Young Scientists. The work of M.E. and K.O. is also supported by the Research Fellowships of the Japan Society for the Promotion of Science for Research Abroad.

\section{References}

[1] A. Achucarro and T. Vachaspati, Phys. Rept. 327, 347 (2000) [arXiv:hep-ph/9904229]; R. Jeannerot, J. Rocher and M. Sakellariadou, Phys. Rev. D 68, 103514 (2003) arXiv:hep-ph/0308134.

[2] A. Vilenkin and E. P. S. Shellard, Cosmic Strings and Other Topological Defects, Cambridge Univ. Press (1994); M. B. Hindmarsh and T. W. B. Kibble, Rept. Prog. Phys. 58, 477 (1995).

[3] A. Hanany and D. Tong, JHEP 0307, 037 (2003) [arXiv:hep-th/0306150].

[4] R. Auzzi, S. Bolognesi, J. Evslin, K. Konishi and A. Yung, Nucl. Phys. B 673, 187 (2003) arXiv:hep-th/0307287.

[5] A. A. Abrikosov, Sov. Phys. JETP 5 (1957) 1174 [Zh. Eksp. Teor. Fiz. 32 (1957) 1442]; H. B. Nielsen and P. Olesen, Nucl. Phys. B 61 (1973) 45.

[6] M. Eto, Y. Isozumi, M. Nitta, K. Ohashi and N. Sakai, Phys. Rev. Lett. 96, 161601 (2006) arXiv:hep-th/0511088]; M. Eto, K. Konishi, G. Marmorini, M. Nitta, K. Ohashi, W. Vinci and N. Yokoi, Phys. Rev. D 74, 065021 (2006) arXiv:hep-th/0607070.

[7] M. Eto, Y. Isozumi, M. Nitta, K. Ohashi and N. Sakai, J. Phys. A 39, R315 (2006) arXiv:hep-th/0602170. 
[8] M. Eto, K. Hashimoto, G. Marmorini, M. Nitta, K. Ohashi and W. Vinci, Phys. Rev. Lett. 98, 091602 (2007) arXiv:hep-th/0609214.

[9] M. Shifman and A. Yung, Phys. Rev. D 70, 045004 (2004) arXiv:hep-th/0403149]; A. Hanany and D. Tong, JHEP 0404, 066 (2004) arXiv:hep-th/0403158]. M. Eto, Y. Isozumi, M. Nitta, K. Ohashi and N. Sakai, Phys. Rev. D 72, 025011 (2005) arXiv:hep-th/0412048.

[10] D. Tong, "TASI lectures on solitons," arXiv:hep-th/0509216; M. Shifman and A. Yung, "Supersymmetric Solitons and How They Help Us Understand Non-Abelian Gauge arXiv:hep-th/0703267.

[11] L. Ferretti, S. B. Gudnason and K. Konishi, Nucl. Phys. B 789, 84 (2008) arXiv:0706.3854 [hep-th]].

[12] Y. Isozumi, M. Nitta, K. Ohashi and N. Sakai, Phys. Rev. D 71, 065018 (2005) arXiv:hep-th/0405129]; Phys. Rev. Lett. 93, 161601 (2004) arXiv:hep-th/0404198]; Phys. Rev. D 70, 125014 (2004) [arXiv:hep-th/0405194].

[13] M. Shifman and A. Yung, Phys. Rev. D 73, 125012 (2006) arXiv:hep-th/0603134; M. Eto, J. Evslin, K. Konishi, G. Marmorini, M. Nitta, K. Ohashi, W. Vinci and N. Yokoi, Phys. Rev. D 76, 105002 (2007) [arXiv:0704.2218 [hep-th]].

[14] M. Eto, L. Ferretti, K. Konishi, G. Marmorini, M. Nitta, K. Ohashi, W. Vinci and N. Yokoi, Nucl. Phys. B 780, 161 (2007) arXiv:hep-th/0611313.

[15] M. Eto, T. Fujimori, M. Nitta, K. Ohashi, K. Ohta and N. Sakai, Nucl. Phys. B 788, 120 (2008) arXiv:hep-th/0703197.

[16] D. Dorigoni, K. Konishi and K. Ohashi, arXiv:0801.3284 [hep-th] (2008).

[17] M. Eto, T. Fujimori, S. B. Gudnason, K. Konishi, M. Nitta, K. Ohashi and W. Vinci, in preparation.

[18] M. A. Luty and W. Taylor, Phys. Rev. D 53, 3399 (1996) arXiv:hep-th/9506098.

[19] P. Cvitanovic, Phys. Rev. D 14, 1536 (1976). 